\title{
Genetic associations between tuberculosis and economically important performance traits in Irish Holstein Friesian dairy cows
}

\section{L Bermingham ${ }^{1}$, S J More ${ }^{2}$, M Good $^{3}$, A R Cromie ${ }^{4}$, I M Higgins ${ }^{2}$, D P Berry ${ }^{1}$}

${ }^{1}$ Moorepark Production Research Centre, Fermoy, Co. Cork, Ireland, ${ }^{2}$ Centre for Veterinary Epidemiology and Risk Analysis, UCD School of Agriculture, Food Science and Veterinary Medicine, University College Dublin, Belfield, Dublin 4, Ireland, ${ }^{3}$ Department of Agriculture, Fisheries and Food, Kildare St, Dublin 2, Ireland, ${ }^{4}$ The Irish Cattle Breeding Federation, Bandon, Co. Cork, Ireland Email: mbermingham@hotmail.com

Introduction Mycobacterium bovis is the principal agent of bovine tuberculosis (bTB). A recent study has demonstrated genetic variation exists among Holstein-Friesian dairy cattle for resistance to M. bovis infection (Bermingham et al. 2009). The objective of this study was to estimate the genetic associations between susceptibility to M. bovis infection and economically important traits.

Materials and methods The single intradermal comparative tuberculin test (SICTT) is used as a measure of susceptibility of cows to $M$. bovis infection. The test involves injecting $M$. bovis-purified protein derivative (PPD) into the neck of each animal, and comparing the reaction induced to that produced by $M$. avium-PPD (a measure of sensitisation to environmental mycobacteria). Susceptibility to $M$. bovis-PPD responsiveness was dichotomised as standard reactor (a $M$. bovis-PPD reaction $4 \mathrm{~mm}$ or greater than the $M$. avium-PPD reaction) or nonreactor (a $M$. bovis-PPD reaction equal to the M. avium-PPD reaction). National SICTT records between November 2000 and December 2007 were available for inclusion in the analysis. Cows that calved outside the normal age for a given parity, that had inconclusive SICTT results, or that moved into the herd within six weeks of the SICTT (it takes three to six weeks to develop a positive reaction to the test post infection) were discarded. Following edits, only episodes (herd restrictions initiated by two or more standard reactors [one of which was home bred], and terminated by two consecutive clear herd tests) with at least one standard reactor and ten or more tested cows were retained; 17,178 M. bovis-PPD responsiveness records from 598 episodes remained. Data on first to third parity 305-day milk, fat, and protein yield, somatic cell score (SCS), calving interval (CI), first parity body condition score (BCS), as well as survival from parity 1 to 2,2 to 3 and 3 to 4 were extracted from the Irish Cattle Breeding Federation database for cows calving between 1985 and 2007. Cows with an age at calving more than 22 months from the parity median, and herd-year-season and paternal half sib groups with less than five records were removed. Following edits, 105,064 (with 2,185 M. bovis infection records) cows had information on production, 112,337 (with 2,389 bTB records) had information on CI, 104, 044 (with 2,895 bTB records) had information on survival, and 57,250 (with 354 bTB records) had information on BCS. Genetic and residual (co)variance components between susceptibility to $M$. bovis-PPD responsiveness and performance were estimated using bivariate linear-linear (LLSM) and threshold-linear (TLSM) sire models in ASREML (Gilmour et al. 2009). The likelihood ratio test of nested models was used to determine whether the correlations differed significantly from zero. The perfomance data were randomly split by herd-year-season contemporary group and correlations re-estimated, to assess the repeatability of correlations.

Results Heritability (standard errors in parentheses) for susceptibility to M. bovis-PPD responsiveness estimated from the threshold model was $0.12(0.024)$. Susceptibility to $M$. bovis-PPD responsiveness was positively genetically correlated with second parity fat production and BCS, and negatively genetically correlated with first parity SCS and survival from parity 3 to 4. Similar correlations were obtained from the LLSM and TLSM and from both sub-sets of data.

Table 1 Genetic correlations (standard errors in parentheses) from linear-linear models between susceptibility to M. bovisPPD responsiveness and economically performance

\begin{tabular}{llll}
\hline \hline Trait & Parity 1 & Parity 2 & Parity 3 \\
\hline Milk yield & $0.23(0.14)$ & $0.24(0.14)$ & $0.13(0.16)$ \\
Fat yield & $0.32(0.14)$ & $0.39(0.13)^{*}$ & $0.23(0.15)$ \\
Protein yield & $0.16(0.15)$ & $0.32(0.14)$ & $0.06(0.16)$ \\
Somatic cell score & $-0.34(0.14)^{*}$ & $-0.11(0.15)$ & $-0.14(0.17)$ \\
Calving interval & $-0.07(0.18)$ & $0.00(0.22)$ & $-0.18(0.30)$ \\
Survival & $-0.08(0.22)$ & $-0.17(0.23)$ & $-0.62(0.22)^{*}$ \\
Body condition score & $0.36(0.14)^{*}$ & & \\
\hline \hline
\end{tabular}

*Genetic correlations significantly different from zero.

Conclusions This is the first study to estimate genetic correlations between susceptibility to $M$. bovis infection and performance. The results from this study suggest that selection for increased survival may indirectly reduce, while selection for reduced SCS and increased fat production and BCS may increase susceptibility to $M$. bovis infection within the national Holstein Friesian dairy herd. However, future work on independent data that corroborates these finding is required before definitive conclusions can be drawn.

Acknowledgements Financial support from ERAD is gratefully acknowledged

\section{References}

Bermingham, M.L., More, S.J., Good, M., Cromie, A.R., Higgins, I.M., Brotherstone, S., and Berry, D.P. 2009. Journal of Dairy Science 92, 3447-3456.

Gilmour, A.R., Cullis, B.R., Welham, S.J., and Tompson, R. 2009. ASReml reference manual, $2^{\text {nd }}$ edition. 